\title{
Using the Health Belief Model, Could the Use of Pedometers Encourage Physical Activity in Adults with Sedentary Occupations and Associate to a Reduction in Obesity and Weight Gain?
}

\section{Areaka Luke-Brinson*}

School of Nursing and Health Sciences, Capella University, USA

"Corresponding author: Areaka Luke-Brinson, School of Nursing and Health Sciences, Capella University, USA, Tel: +7069416452; E-mail: aluke5@capellauniversity.edu

Received date: April 05, 2018; Accepted date: April 09, 2018; Published date: April 16, 2018

Copyright: (C) 2018 Luke-Brinson A. This is an open-access article distributed under the terms of the Creative Commons Attribution License, which permits unrestricted use, distribution, and reproduction in any medium, provided the original author and source are credited.

\section{Abstract}

Aim: The aim of this paper is to examine how the Health Belief Model can be used to demonstrate the benefits of pedometer use for sedentary working adults to aid in decreasing obesity and weight gain.

Introduction: Pedometers have grown to be a popular tool to track steps resulting in demonstrating the number of calories burned from the number of steps tracked.

Methods: There will also be a breakdown of pedometer use in different circumstances that document and demonstrate the benefits of using pedometers to reduce a sedentary lifestyle. Using the Health Belief Model, the concepts that will be analyzed will be: individual perceptions; modifying actions; and likelihood of change.

Conclusion: Pedometer use could begin the process of lowering the prevalence of ailments that can lead to death.

Keywords: Physical activity; Sedentary lifestyle; Obesity; Adults

\section{Introduction}

With a high demand from the workforce along with obligations to fulfill outside of work, adults hardly have enough time in the day to perform enough physical activity to manage a healthy weight. Many working adults have deskbound professions and have such a large workload that they do not get a chance to walk away from their work desks for extended periods of time. Pedometers have grown to be a popular tool to track steps resulting in demonstrating the number of calories burned from the number of steps tracked. The reason for this admiration is because these devices make it easier to track the physical activity of walking, jogging, and running. These devices count steps taken daily so that this activity can be chronicled weekly [1]. Having a visual image of steps along with calories burned can encourage physical activity. A cardiac rehab study states that "pedometers made steps visible, leading to a conscious, tailored activity supporting patient autonomy and independency" [2]. Some of these devices can give hourly reminders to move when no physical activity has been detected. This can grant the individual attention on lack of physical activity and encourage them to move more by taking more steps daily and burning more calories. This can also be associated with behavior change and better weight management and decrease weight gain and obesity.

\section{Methods}

\section{Perceived seriousness, severity, and threat}

Obesity is a rising issue within the United States that is caused by a variety of factors which include improper diet and lack of physical activity. One study mentions that a rising percentage or our population is overweight or obese with several ailments and that these individuals are at a higher risk for hostile health outcomes [1]. Obesity correlates with metabolic syndrome, and metabolic syndrome leads to a raised hazard of cardiovascular disease and type two diabetes mellitus [3]. The aim of this paper is to examine how the Health Belief Model can be used to demonstrate the benefits of pedometer use for sedentary working adults to aid in decreasing obesity and weight gain. There will also be a breakdown of pedometer use in different circumstances that document and demonstrate the benefits of using pedometers to reduce a sedentary lifestyle. The concepts that will be analyzed will be: individual perceptions; modifying actions; and likelihood of change (Figure 1).

\section{Modifying factors and cues to action: Effects of pedometer use on attitude and behavior}

Currently as fulltime employed adults, it could be challenging to maintain or to incorporate physical activity, and this can be discouraging. Most of the population of occupied adults desire to incorporate some type of physical activity outside of the workplace but may not have the motivation nor may not know how to follow up on incorporating it, thus leading to a negative attitude toward physical activity. An article from an observational cohort study documents that "overweight and obese persons in Iran with type 2 diabetes mellitus identified barriers to physical activity that included negative attitude toward physical activity, discouragement, physical problems, cost and environmental factors" [3]. There is study evidence that document the use of pedometers and their means of promoting aerobic fitness [4]. Another study states that most recent public health approvals state that the average adult to complete 10,000 steps per day [5]. It is practical 
Citation: Luke-Brinson A (2018) Using the Health Belief Model, Could the Use of Pedometers Encourage Physical Activity in Adults with Sedentary Occupations and Associate to a Reduction in Obesity and Weight Gain?. J Health Educ Res Dev 6: 257. doi: $10.4172 / 2380-5439.1000257$

Page 2 of 2

that having a visual cue such as pedometer devices like watches or smartphones can aid in keeping track with the number of steps. Having access to this visual cue could make it easier in assisting in positive behavior modification of increasing physical activity. This access can also alter the attitudes that individuals have toward physical activity by encouraging the act of setting goals. A study concludes that pedometer use demonstrates self-monitoring which can also create a positive attitude towards increasing physical activity and developing a change in behavior [6,7]. Even though the recommendation is 10,000 steps daily, this can encourage adults to meet this recommendation or surpass this standard.

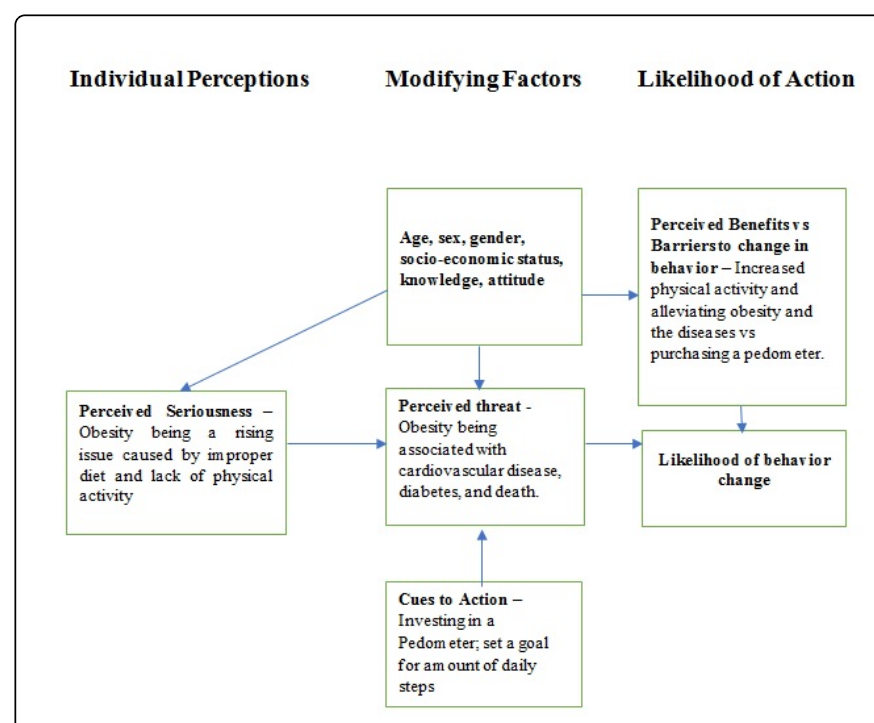

Figure 1: Health belief model for pedometer use.

\section{Perceived benefits vs barriers to change: Effects of pedometer use on morbidity and mortality}

Pedometer use could begin the process of lowering the prevalence of ailments that can lead to death. A study mentions that patients who have musculoskeletal complications demonstrate enhancements in disability, strength, and pain scores just by adding 1,950 steps per day
[3]. The same study indicates that increased number of steps was associated with decreased all-cause rates in death [3].

\section{Conclusion}

\section{Likelihood of behavior change}

In inference, obesity is a growing public health concern in the United States and can lead to other ailments. Therefore, pedometer use can be useful and aiding in alleviating obesity. Once the individual observes their sedentary lifestyle, they can analyze the severity and risks of the public health concern. At times, it may take scare tactics such as gaining knowledge of the matter and the underlying ailments. This information along with the action indications can create a positive attitude to initiate modification in behavior.

\section{References}

1. Takahashi P, Quigg SM, Croghan IT, Schroeder DR, Ebbert JO (2016) Effect of pedometer use and goal setting on walking a functional status in overweight adults with multimorbidity: a crossover clinical trial. Clin Interv Aging 11: 1099-1106.

2. Thorup CB, Gronkjaer M, Spindler H, Andreasen JJ, Hansen J, et al. (2015) Pedometer Use as Motivation for Physical Activity in Cardiac TeleRehabilitation. Int J Integrat Care 15: 1-3.

3. Joven MH, Croghan IT, Quigg SM, Ebbert JO, Takahashi PY (2017) Predictors of sedentary status in overweight and obese patients with multiple chronic conditions: a cohort study. Pragmat Obs Res 8: 203-209.

4. Lystrup R, West GF, Ward M, Hall J, Stephens M (2015) Exploring the Impact of a Pedometer on Body Composition and Physical Fitness in a Cohort of US Military Medical Students: A Pilot Study. Mil Med 180: 23-25.

5. Norman GJ, Heltemes KJ, Heck D, Osmick MJ (2016) Employee Use of Wireless Physcial Activity Tracker Within Two Incentive Designs at One Company. Popul Health Manag 19: 88-94.

6. Avery L, Flynn D, Dombrowski SU, Van Wersch A, Sniehotta FF, et al. (2015) Successful behavioural strategies to increase physical activity and improve glucose control in adults with Type 2 diabetes. Diabet Med 32: 1058-1062.

7. Sullivan AN, Lachman ME (2017) Behavior Change with Fitness Technology in Sedentary Adults: A Review of the Evidence for Increasing Physical Activity. Front Public Health 4: 289. 